\title{
After bioethics and towards virtue?
}

\author{
Peter D Toon St Bartholomew's Hospital Medical School, London
}

\begin{abstract}
Author's abstract
The place of philosophical medical ethics in medical education and clinical practice has recently been questioned. Although partially valid, the criticisms do not warrant abandoning the enterprise. Instead a reappraised model, based on Aristotelean concepts of intellectual and moral virtue is suggested.
\end{abstract}

There appears to be growing disquiet about the achievements and future of medical philosophy or bioethics. After a period of rapid and sustained growth in movements of thought a reaction is typical. What is unusual is that the backlash has come, not from those threatened by the movement, but from some of those most involved in its development.

Thus the fournal of Medicine and Philosophy has devoted a whole issue recently (1) to assessing the progress of the field. In the introduction (2) the editors voice their surprise that rather than papers from philosophers outside the field known to be critical of it, they have received critical papers by authors themselves active in medical philosophy. Even more remarkable is a recent article in which Seedhouse (3) directs his vitriol not at medical practitioners, as is his wont, but at medical ethicists. $\mathrm{He}$ comes to the conclusion that the sooner they cease to exist the better, a view surprising in one who earns his living as a medical philosopher.

The common strands in various critiques seem to be that:

1) standards of academic scholarship in bioethics are often poor.

2) philosophical analysis cannot solve the problems of ethical decision-making in medicine.

3) if we wish to have morally better decisions made in medicine then instead of training doctors intellectually in applied ethics, we need to make them more morally virtuous.

\section{Key words}

Medical education; ethics; philosophy of medicine; virtue.
These criticisms have some truth but do not justify writing off medical philosophy. Rather we need to address its weaknesses and consider how we might overcome them.

Academic standards in any field vary. Some medical ethics is good, some is bad and most is moderate - useful, interesting clarification of issues and mildly original ideas which add a little to our understanding of the world. Whilst the low esteem in which it is held both by philosophers who view applied philosophy as too tainted by the world to be rigorous, and by medical academics suspicious of any activity which cannot produce numerical data, may deter some of the most able, it is not lack of academic ability that is mainly responsible for poor work.

The major threat is the bad faith of those who enter the field with a hidden agenda. Many bioethicists have an axe to grind; most often a Kantian axe labelled autonomy, but smaller axes of interprofessional rivalry and antiprofessionalism are also common. Whilst grinding axes is a perfectly respectable occupation, it must not be cloaked in a pretence of academic neutrality. Impartial analysis must be clearly distinguished from the support of a particular position, and presuppositions and prejudices must be openly and honestly stated.

Disenchantment with the results of medical philosophy arises largely because too much has been expected and claimed for bioethics. An example of the result of placing an excessive burden on a concept unable to sustain it is what Clouser and Gert (4) call principlism; the notion that beneficence, autonomy, justice, and non-maleficence could solve ethical problems rather than be a useful framework for clarifying them.

It is foolish to believe that a knowledge of moral philosophy or an ethical analysis makes a difficult moral decision easy, anymore than knowledge of physiology and pathophysiological analysis makes a complex clinical case simple to diagnose or to prognosticate. In both cases ars longa vita brevis. What sound training in philosophical analysis can do for moral problems is exactly parallel to what training in clinical sciences can do for the diagnostic problem: ie provide a framework in which choices can be 
organised and evaluated logically, avoiding conclusions not justified by the evidence and decisions made on irrelevant grounds.

Clinical teachers have all seen the inexperienced student listen to a murmur and, without careful attention to what she is hearing, leap to an incorrect conclusion as to its meaning. Those without rigorous training are prone to jump to conclusions without thinking through their decisions. The intellectual discipline to stop and dissect unorganised data, attending systematically to each aspect, selecting appropriately and linking them together, and then considering carefully the deductions one can and cannot reasonably make from them is difficult both to teach and to learn, yet even experienced clinicians would admit that most of their mistakes come from failure to think in a disciplined way.

However experienced they might be, doctors most of the time do not work from first principles, but heuristically, solving problems by a hypotheticodeductive method which bypasses most of the analytical thinking taught to students. There are however three important differences between the experienced and the neophyte. Firstly the experienced clinician has the ability to check the heuristically reached conclusion against the strict canons of clinical analysis; secondly in hard cases when the heuristic method fails, the clinician can 'fall back' on the more time-consuming process of systematic analysis. Thirdly the clinician learns, in ways we do not fully understand, to reach sound conclusions without consciously going through the process of logical justification. This we refer to as sound clinical judgement. Unlike clinical analysis we find this hard to teach, though we can recognise it in those who possess it.

In an exactly parallel way the doctor untrained in moral philosophy is prone to jump to ethical conclusions on the basis of gut feeling, without proper consideration of the issues involved. Illogical leaps are made, judgement is inappropriately swayed by emotive language, and inconsistent positions are adopted.

To avoid such irrationality students must learn to analyse the ethical problems which they face logically and to justify their conclusions. Seedhouse (1) is right in saying that this is not sufficient. However, we cannot rely on a vague confidence that a new generation of moral generalists will emerge, as he suggests. This will lead to a return to doctors dressing their prejudices up as professional judgements, with no one to call them to account. Rather we need to learn how to teach the equivalent of clinical judge- ment (which Aristotle would refer to as an intellectual virtue) in the moral sphere: moral virtue.

Producing virtuous doctors is not the same as training them in the intellectual skills of philosophical analysis, but we cannot abandon ethical analysis. Just as the doctor needs the structures of clinical analysis to fall back on and to check her heuristically derived hypotheses, so the virtuous doctor will need moral theory as a lifeboat in cases of difficulty.

Medical educators are currently trying to teach clinical judgement, so that we no longer have to wait for it to emerge by an unseen and ill-understood process. So too medical ethics needs to consider how to cultivate moral virtue in medical practitioners. This will be both harder and easier than the clinician's task. Harder because it will involve breaking down artificial barriers between academic fields and other aspects of human life which are deeply entrenched in our post-Enlightenment culture. Easier because once those barriers are broken the structures for teaching and learning moral virtue already exist, in the work of psychologists, theologians and mystics as well as philosophers.

Peter D Toon is a General Practitioner in Hackney, London and Honorary Lecturer, Departments of General Practice and Primary Care, and Human Science and Medical Ethics, Medical Colleges of St Bartholomew's and the Royal London Hospitals, Charterhouse Square, London EC1.

\section{Acknowledgement}

This paper was written during the tenure of a Visiting Fellowship, Bioethics Research Unit, University of Otago Medical School, Dunedin, New Zealand. Thanks are due to Professor Alistair Campbell and the staff of his department for their hospitality, and to the Royal College of General Practitioners and the Department of Health, UK for grant support.

\section{References}

(1) Symposium: Philosophical critiques of bioethics. fournal of medicine and philosophy 1990: April issue.

(2) Anonymous [editorial]. Philosophical critiques of $\mathrm{N}$ bioethics. Fournal of medicine and philosophy 1990; 15, 2: 121 .

(3) Seedhouse D. Against medical ethics; a philosopher's view. Medical education 1991; 25, 2: 280-282.

(4) Clouser K D, Gert B. A critique of principlism. Fournal of medical philosophy 1990; 15, 2: 219-236. 